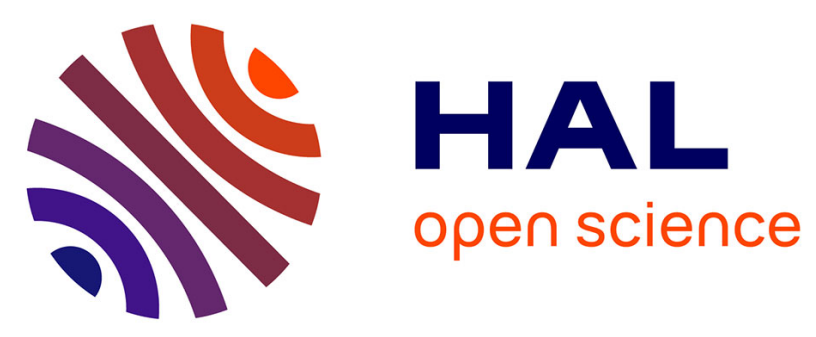

\title{
Short Note Hydrophone Calibration at Very Low Frequencies
}

Cécile Joubert, Guust Nolet, Alexey Sukhovich, Anthony Oge, Jean-François Argentino, Yann Hello

\section{- To cite this version:}

Cécile Joubert, Guust Nolet, Alexey Sukhovich, Anthony Oge, Jean-François Argentino, et al.. Short Note Hydrophone Calibration at Very Low Frequencies. Bulletin of the Seismological Society of America, 2015, 105 (3), pp.1797-1802. 10.1785/0120140265 . insu-01172179

\section{HAL Id: insu-01172179}

\section{https://hal-insu.archives-ouvertes.fr/insu-01172179}

Submitted on 7 Jul 2015

HAL is a multi-disciplinary open access archive for the deposit and dissemination of scientific research documents, whether they are published or not. The documents may come from teaching and research institutions in France or abroad, or from public or private research centers.
L'archive ouverte pluridisciplinaire HAL, est destinée au dépôt et à la diffusion de documents scientifiques de niveau recherche, publiés ou non, émanant des établissements d'enseignement et de recherche français ou étrangers, des laboratoires publics ou privés. 


\title{
Short Note \\ Hydrophone Calibration at Very Low Frequencies
}

\author{
by Cécile Joubert," Guust Nolet, Alexey Sukhovich, Anthony Oge, ${ }^{\dagger}$ \\ Jean-François Argentino, and Yann Hello
}

\begin{abstract}
We propose a new method to study the response of a hydrophone at very low frequencies. In our method, the hydrophone is placed in a calibration chamber filled with water and, by instantaneously changing the water height, an abrupt pressure increase of about $1000 \mathrm{~Pa}$ is produced. The pressure variation mathematically corresponds to an input signal close to a step function. The response is recorded after filtering and digitizing so that we obtain the response of the complete system.

We also report on the development of an automatic method to determine the number of poles and zeros and their values that describe the observed response. We apply our method to the RAFOS II hydrophone, used on the Mobile Earthquake Recorder in Marine Areas by Independent Divers (MERMAID) floats. As an illustration of the method, an instrumental response in terms of poles and zeros is used to correct seismograms from the 7 April $2014\left(M_{\mathrm{w}} 4.8\right)$ Barcelonnette earthquake, recorded by three MERMAIDs deployed in the Mediterranean Sea, and to express the observed signals in pascals.
\end{abstract}

\section{Introduction}

Knowing the instrument response is important in quantitative seismology. Recently, hydrophones mounted on oceanographic Mobile Earthquake Recorder in Marine Areas by Independent Divers (MERMAID) floats, cruising at depths down to $2000 \mathrm{~m}$ in the Mediterranean Sea and Indian Ocean, have begun to record $P$ waves from teleseismic and local earthquakes. The MERMAID float was developed to record teleseismic arrival times from strong earthquakes and to improve the knowledge of the dynamics of the mantle, more specifically by imaging the hotspot-rich mantle in the southern hemisphere, where the lack of land-based seismic stations results in poorly resolved global tomographic models (Simons et al., 2006, 2009; Hello et al., 2011). MERMAIDs have also demonstrated the ability to record weak local and regional events. As an example, Figure 1 shows the $P$ phases recorded by three MERMAIDs of an earthquake that occurred on 7 April 2014 in Barcelonnette (France), with a magnitude of $M_{\mathrm{w}}$ 4.8. Global Positioning System positions of the floats that detected this earthquake are shown in Figure 2 (float position is determined after it surfaces to transmit the recording).

To correlate $P$ arrivals in different instruments, or simply to estimate the amplitude correctly, it is necessary to correct

\footnotetext{
* Now at Université Nice Sophia Antipolis, CNRS, IRD, Observatoire de la Côte d'Azur, Géoazur UMR 7329, 250 rue Albert Einstein, Sophia Antipolis 06560 Valbonne, France.

'Now at ONIRIS, CNRS, GEPEA UMR 6144, 44322 Nantes, France.
}

for the instrument response. It is challenging to calibrate hydrophones at such very low frequencies $(<2 \mathrm{~Hz})$ (Giangreco, 1997). Various methodologies, such as reciprocity or substitution (Bobber, 1988; Smith and Bacon, 1990; Radulescu et al., 2003; Koch and Wilkens, 2004; Veledar, 2009), have been proposed for the kilohertz and megahertz frequency range but few for very low frequencies. Methodologies developed by Levin (1973) and McEachern (1984) propose a dynamic calibration with a hydrophone displacement in a pressure field, whereas Golenkov and Pavlov (1972) and Zalesak (1999) propose a static one, in which the pressure itself is variable. In the Levin (1973) method, a hydrophone is placed along an inflexible vertical axis that is connected to a wheel and a motor. The wheel rotation leads to a vertical oscillation motion of the hydrophone in water, and the signal is recorded by a voltmeter. In the McEachern (1984) method, a hydrophone to be calibrated, as well as a reference hydrophone, are connected to a voltmeter and fixed along a rocker arm, which is also connected to a motor. The motor rotation leads to a vertical oscillation motion of the hydrophones, and the recorded amplitudes are compared. Golenkov and Pavlov (1972) propose a similar method in which a hydrophone is immersed in a water tank connected to a vibrating chamber by means of a flexible cable. This open vibrating chamber is filled with water. The chamber vibrations are transmitted to the hydrophone, and the recorded signals are studied. The Zalesak (1999) method is an extension of the reciprocity method at 


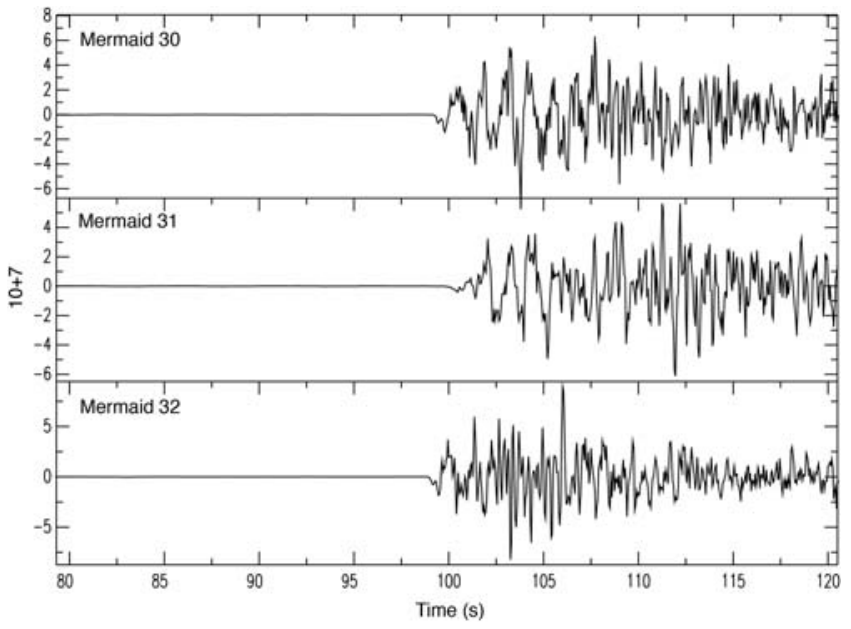

Figure 1. Raw digitizer output of the Barcelonnette earthquake $\left(M_{\mathrm{w}} 4.8\right)$ recorded by three Mobile Earthquake Recorder in Marine Areas by Independent Divers (MERMAIDs) on 7 April 2014. The time axis is in seconds and begins at 19:25:45 UTC. The amplitude axis is in counts.

high frequencies. The hydrophone to be calibrated, a projector, and a transducer are placed in a closed calibration chamber. The hydrophone response is determined by measuring the electrical impedance of each pair of transducers.

In this study, we test a new method for hydrophone calibration at very low frequencies. To study the hydrophone response, the hydrophone is subjected to an abrupt pressure variation of about $1000 \mathrm{~Pa}$, which is close to the saturation of the electronics but still linear, and the output signal is analyzed in terms of poles and zeros. Rather than measuring the response of the hydrophone itself in volts, we measure the response of the complete system, including electronics, filtering, and digitizing stages in counts, which enables us to correct the digital signals for the instrument response. In our method, the hydrophone is rigidly fixed in a calibration chamber filled with water. The pressure variation is created by opening a solenoid valve positioned between the chamber and a vertical tube that is also filled with water (Fig. 3). The water level in a tube ( $10 \mathrm{~cm}$ in our case) controls the amount of the induced pressure variation. The sudden pressure increase generates an input signal close to a step function, though we introduced a short, linear rise of duration $t_{\tau}$ (equation 3). We applied our method to calibrate a RAFOS II hydrophone, developed by Teledyne Benthos and used in the MERMAID. For teleseismic $P$ waves, the frequency band of interest is between 0.01 and about $2 \mathrm{~Hz}$. The RAFOS II frequency response is flat between $5 \mathrm{~Hz}$ and $10 \mathrm{kHz}$, but no factory data are supplied for frequencies under $5 \mathrm{~Hz}$.

\section{Experimental Details}

As shown in Figure 3, the hydrophone is attached to the upper part of a cylindrical calibration chamber. The calibration chamber is carefully filled with water, and we make sure that no air bubbles are present. The impermeability at the

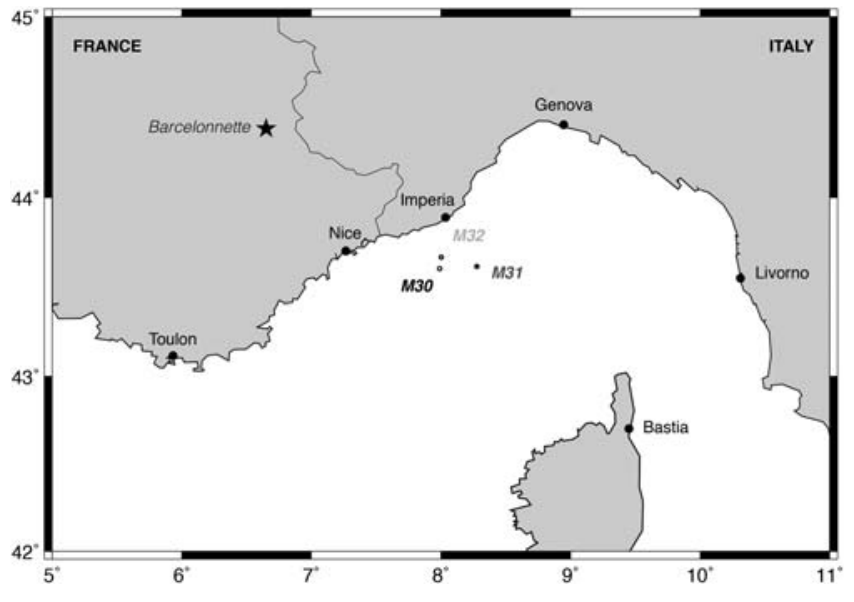

Figure 2. Locations of the three MERMAID floats (M30, M31, and M32) during their transmission of the Barcelonnette earthquake records.

base of the hydrophone is accomplished through an O-ring. The upper part of the chamber is also sealed to the lower part through a circular O-ring and three screws at $120^{\circ}$ from each other, which ensure the chamber is completely sealed.

To the upper part of the chamber, we mounted a solenoid valve, which controls the contact between the water in the calibration chamber and an additional water column placed above the solenoid valve. The valve is connected to a stable power supply. Using a water density of $\rho=998 \mathrm{~kg} / \mathrm{m}^{3}$, a local gravity acceleration $g=9.804 \mathrm{~m} / \mathrm{s}^{2}$, and $\Delta h=0.1 \mathrm{~m}$, we obtain a pressure increase of $\Delta P=978.4 \mathrm{~Pa}$, for a water column of $10 \mathrm{~cm}$. When we open the valve, the two fluids come into direct contact, which creates a pressure increase detected by the hydrophone. To independently monitor the pressure increase time, a pressure sensor is placed at the base of the chamber (Fig. 3, lower circle). This is used to measure the short time $\left(t_{\tau}<1 \mathrm{~s}\right)$ it takes for the pressure increase to establish itself. This second sensor, however, was too noisy to be used for direct calibration.

Between the solenoid valve and the upper part of the calibration chamber, a purge allows us to flush out the water column before repeating the experiment.

The hydrophone is connected to the data-acquisition card of the MERMAID float by a coaxial cable. The acquisition frequency extends from 0.1 to $10 \mathrm{~Hz}$ with a band-pass filter integrated in the electronic card, and the acquisition sampling frequency of $40 \mathrm{~Hz}$ is the same as used in the MERMAID.

Ensuring reproducibility of signals is an important point, and several difficulties had to be overcome during the method development. A strict protocol was set in place, and a quiet environment is required to limit signal disturbances. At $t=0 \mathrm{~s}$, the data acquisition begins, and the chronometer starts. At $t=1 \mathrm{~min}$, we open the solenoid valve. At $t=2 \mathrm{~min}$, the acquisition stops; and, before $t=3 \mathrm{~min}$, we flush out and adjust the water level in the additional water column for the next experiment. The water level adjustment needs to be precise to produce the desired pressure increase. After $t=6 \mathrm{~min}$, required to 


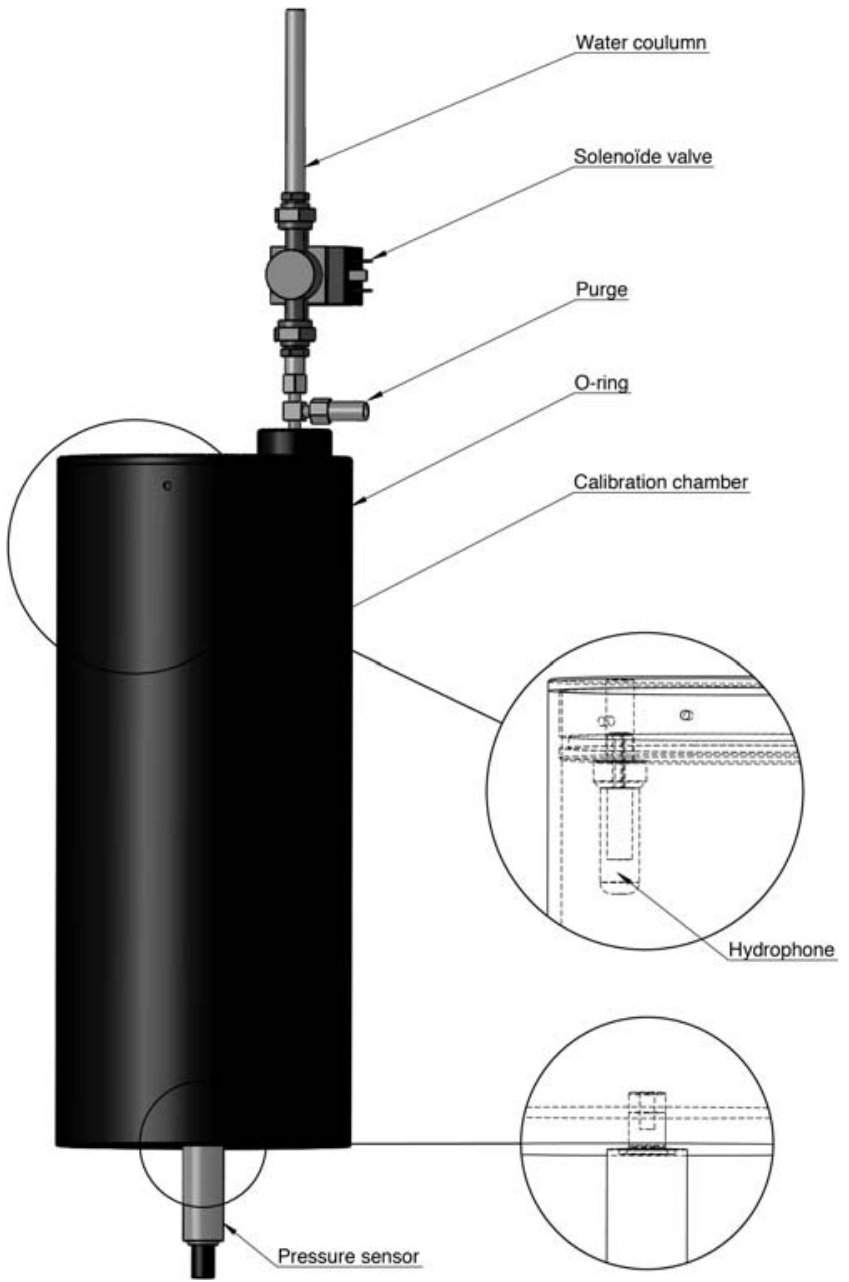

Figure 3. Mechanical setup of the calibration method. The upper and lower enlargements show the hydrophone and the second pressure sensor assemblies, respectively.

allow the hydrophone and the high-pass filter to stabilize, another cycle can begin. Acquisitions took place on several days during May 2014 at different times of the day and also under different temperature conditions to confirm the reproducibility of signals. The measured response has a mean maximum amplitude of $1.54 \pm 0.1310^{8}$ counts. Experiments with a maximum amplitude beyond twice the standard deviation were rejected. A total of 21 response curves were accepted.

\section{Poles and Zeros Determination}

Wielandt (2012) and Ringler et al. (2012) describe methods to determine the poles and zeros of an instrument from an impulse response. Because we were unable to find a simple algorithm to get the poles and zeros from an observed response to a step function in the seismological literature, we describe the interactive method we developed to determine the poles and zeros, which is the basis of the program polezero. The program is written in FORTRAN 90 and uses Generic Mapping Tools for plotting (Wessel and Smith, 1991).
With the Fourier sign convention

$$
H(\omega)=\int_{0}^{\infty} h(t) \exp (i \omega t) d t
$$

the output spectrum $H(\omega)$ is approximated by $n_{p}$ poles and $n_{z}$ zeros as

$$
H(\omega)=A_{0} \frac{\left(i \omega-z_{1}\right)\left(i \omega-z_{2}\right) \ldots\left(i \omega-z_{n_{z}}\right)}{\left(i \omega-p_{1}\right)\left(i \omega-p_{2}\right) \ldots\left(i \omega-p_{n_{p}}\right)} .
$$

The spectrum $H(\omega)$ is Fourier transformed to predict the step response $h(t)$ in the time domain, and the squared misfit with the observed signal $J=\int\left[h(t)-h_{\mathrm{obs}}(t)\right]^{2} d t$ is minimized as a function of $A_{0}$, as well as the poles $p_{i}$ and zeros $z_{i}$.

We give the theoretical development for a step response. For the impulse response, an extra zero $z_{n_{z+1}}=0$ needs to be added.

In the first experiments, we noticed the assumption of a pure step function input led to an underestimate of the response for the highest frequencies; we therefore decided to measure the rise time to a maximum amplitude using the second sensor connected to an oscilloscope, and we allow the influence of a rise time $t_{\tau}$ of the pressure increase (Fig. 4). For a pure step input function of amplitude $P_{0}, F(\omega)=$ $i P_{0} / \omega$. Incorporating a linear rise over time $t_{\tau}$, the input spectrum becomes

$$
\begin{aligned}
F(\omega) & =P_{0} \int_{0}^{t_{\tau}} \frac{t}{\tau} \exp (i \omega t) d t+P_{0} \int_{t_{\tau}}^{\infty} \exp (i \omega t) d t \\
& =P_{0} \frac{\exp \left(i \omega t_{\tau}\right)-1}{\omega^{2} t_{\tau}} .
\end{aligned}
$$

The determination of the poles and zeros, as well as their number, is a highly nonlinear process. It is therefore important to have an acceptable starting guess for the response in terms of poles and zeros. In addition, one is often unsure what the optimal number of poles and zeros might be. To find a good starting set of poles and zeros, we distinguish two cases for a first approximation of the response to a pure step function, neglecting $t_{\tau}$ : the underdamped case uses

$$
h(t)=(A+B t) \exp (-\alpha t)
$$

and the critical or overdamped case uses

$$
h(t)=A \exp (-\alpha t)
$$

In the underdamped case (Fig. 4, lower curve), we parametrize a stack of recorded signals in terms of a simplified response with an overshoot, to find a first starting guess for one zero and two poles:

$$
h(t)=(A+B t) \exp (-\alpha t)
$$

of which the Fourier transform is 


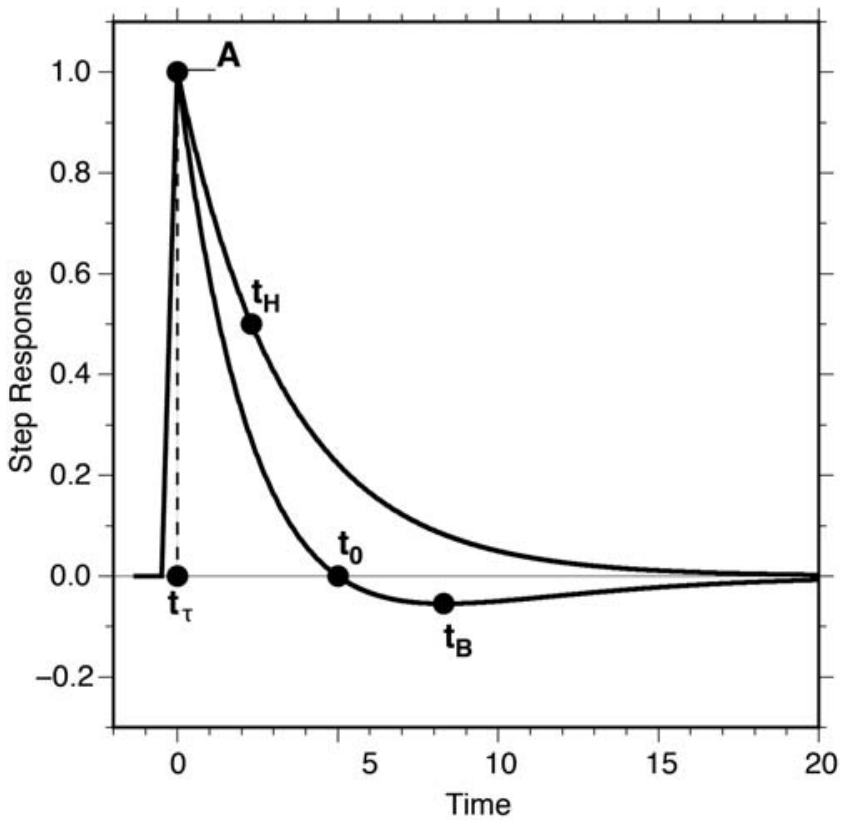

Figure 4. Parameters of the two responses considered in the article. The underdamped response is described by a maximum amplitude $A$; the time of the first zero crossing $\left(t_{0}\right)$ and minimum amplitude $\left(t_{B}\right)$ are included. The overdamped response is described by a maximum amplitude $A$ and the $t_{H}$ at which the amplitude of the response decreases to the value of $A / 2$. In both cases, we also take into account the time of the pressure increase $t_{\tau}$ (measured independently by the second pressure sensor, in the underdamped case).

$$
H(\omega)=-A \frac{i \omega-\alpha-B / A}{(i \omega-\alpha)^{2}},
$$

from which we recognize one real zero at $i \omega=\alpha+B / A$ and two real poles at $\alpha$.

Figure 4 shows the important parameters used for the estimation of the first set of poles and zeros, assuming a pure step function input. We estimate $A$ by measuring the maximum of the response. An estimate for $B$ is subsequently found from the first zero crossing:

$$
B=-\frac{A}{t_{0}}
$$

The time of the minimum amplitude in the response gives an estimate of $\alpha$. Setting the derivative of $h(t)$ to zero gives

$$
\alpha=\frac{B}{A+B t_{B}},
$$

in which $t_{B}$ is defined by $h^{\prime}\left(t_{B}\right)=0$. The two poles and one zero defined by these estimates of $A, B$, and $\alpha$ are used as starting values for the nonlinear optimization.

Because we are not sending a pure step input function, we allow for the influence of a rise time $t_{\tau}$ for the pressure increase. $t_{\tau}$ is read from the second pressure sensor, which is sampled at very high frequency on the oscilloscope.

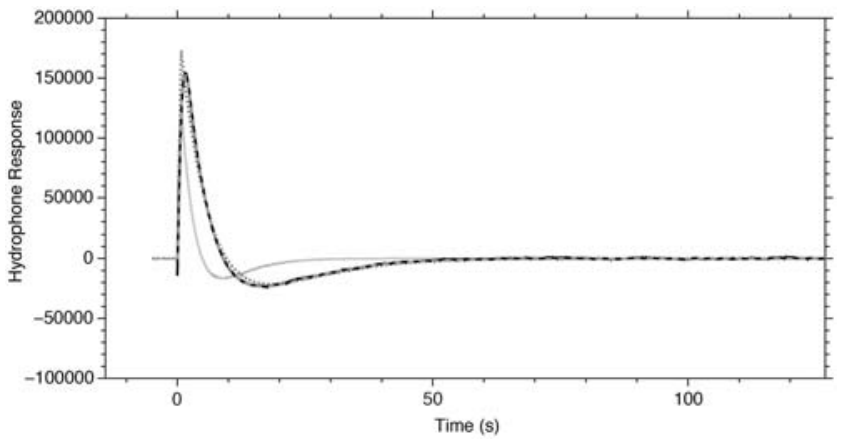

Figure 5. The step response of the MERMAID system (black curve) and the final fit for four poles and three zeros (dashed gray curve). The starting fit (using equation 7 , with two poles and one zero) and the result of the first iteration are given by the light gray and the dotted gray curves, respectively.

If the system is critically damped or overdamped and does not show an overshoot (Fig. 4, upper curve), a simple exponential decay with $B=0$ is assumed, but we maintain $n_{p}=2$ and $n_{z}=1$ even though in that case one pole and one zero are equal to $\alpha$ and could be divided out; instead, we allow the first iteration of optimization to change them into values that are different from each other. In this case, we estimate the starting value $\alpha$ from the half time $t_{H}$ in which the response has decreased to $A / 2$ :

$$
\alpha=\frac{-\ln 0.5}{t_{H}}=\frac{0.693}{t_{H}} .
$$

Once we obtain the optimum fit for the starting configuration of two poles and one zero (both real), we may proceed by adding a pair of one complex zero and one complex pole that are each identical to $(1+i)$. Because these divide out, the starting fit of the next iteration is the same as the one with fewer poles and zeros. In the case of complex poles, the requirement that the response is real forces the program to add their complex conjugates as well, but these are not independent from their counterpart and thus do not add to the number of parameters in the optimization. In each iteration, we thus increase the number of variables to optimize by four (two real and two imaginary components). We used Powell's method (Press et al., 1992) to converge and determine the number of additional poles and zeros by trial and error. Though somewhat slow, Powell's method was found to be much more robust than a gradient search. In our experience, it always leads to a satisfactory fit, comparable with the noise, in the time domain.

\section{Results}

We determined the poles and zeros for the RAFOS II hydrophone in series with the MERMAID electronic card. We are in the underdamped case. We average the 21 response curves to give the black curve in Figure 5. The response is defined by a maximum amplitude of $A=1.5410^{8}$ counts at $t_{\tau}=0.73 \mathrm{~s}$, a zero crossing time of $t_{0}=4.51 \mathrm{~s}$, and a time 


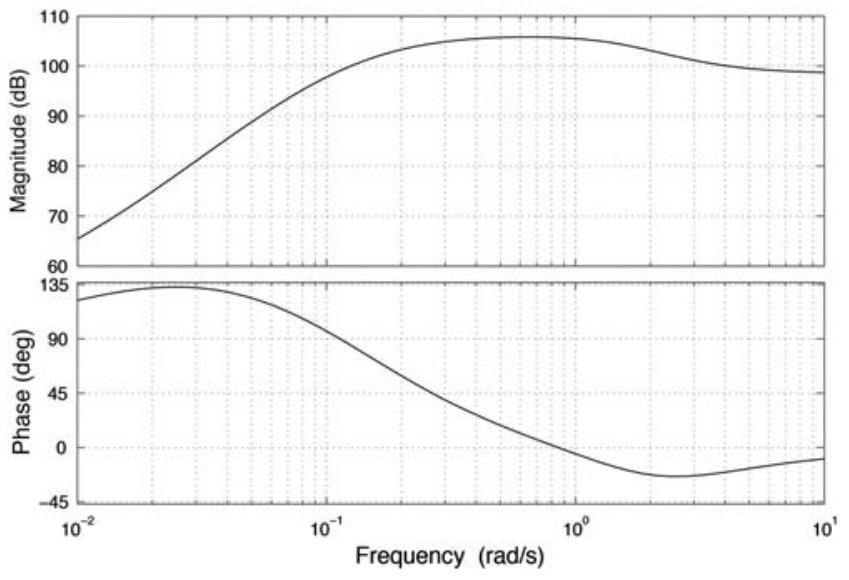

Figure 6. Bode diagram for the poles, zeros, and constant of the studied system.

at minimum signal of $t_{B}=8.52 \mathrm{~s}$. After three iterations, we obtain a misfit of 0.002 , indicated by the thick gray dashed line in Figure 5. The associated poles and zeros for the impulse response (with extra zero added) are listed in Table 1 and illustrated with a Bode diagram in Figure 6 (following the notation and using sign conventions for use with the Seismic Analysis Code, the first and second columns, respectively, represent the real and imaginary parts of the poles and zeros expressed in radians per second and $A_{0}$ in counts/Pa). If another pole and zero pair is added, the misfit does not change the third decimal.

The value of $t_{\tau}$ is determined by the second sensor with a precision better than $0.1 \mathrm{~s}$. The accuracy of the poles and zeros estimates was tested by computing them for three values of $t_{\tau}$. Our best estimate (from 21 experiments) yields $t_{\tau}=0.73 \pm 0.09 \mathrm{~s}$. In Figure 7, we illustrate the instrument correction for the Barcelonnette earthquake recorded by MERMAIDs (Fig. 1). The variability between $t_{\tau}$ values induces a weak influence of $t_{\tau}$ (black curve $t_{\tau}=0.73 \mathrm{~s}$, dashed black curve $t_{\tau}=0.65 \mathrm{~s}$, and dashed gray curve $t_{\tau}=0.80 \mathrm{~s}$ ). Although the response at high frequencies is affected by the values of $t_{\tau}$, the actual effect on the seismogram is minor, even for this case of a regional earthquake with frequencies in excess of $2 \mathrm{~Hz}$. Thus, the Barcelonnette earthquake produced a maximum pressure increase of about $500 \mathrm{~Pa}$ on MERMAID float records.

Table 1

Poles and Zeros for the Impulse Response Determined for the RAFOS II Hydrophone with MERMAID Electronic Card in the Undamped Case

\begin{tabular}{lccr}
\hline \multicolumn{2}{c}{ Poles 4 (rad/s) } & \multicolumn{2}{c}{ Zeros 4 (rad/s) } \\
\hline-0.111545250 & 0.00000000 & 0.00000000 & 0.00000000 \\
-0.152957797 & 0.00000000 & -0.011453878 & 0.00000000 \\
-1.40562248 & -0.882738054 & -2.36022949 & -1.17094541 \\
-1.40562248 & 0.882738054 & -2.36022949 & 1.17094541 \\
\hline
\end{tabular}

Constant $A_{0}=0.8380073 \times 10^{05}$ counts $/ \mathrm{Pa}$.

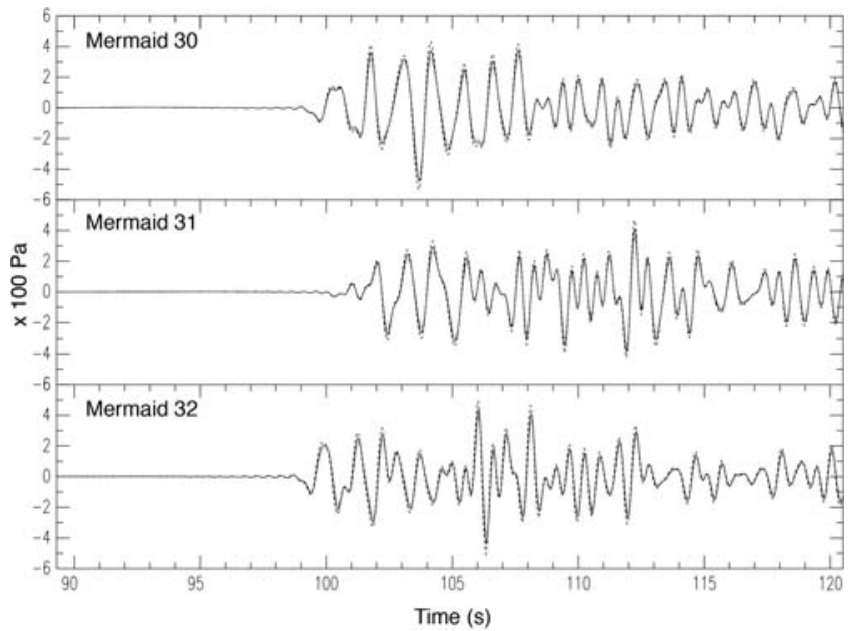

Figure 7. Instrument-corrected MERMAID records of the Barcelonnette earthquake for different values of $t_{\tau}$ : the dashed black signal for $t_{\tau}=0.65 \mathrm{~s}$, the black signal for $t_{\tau}=0.73 \mathrm{~s}$, and the dotted gray signal for $t_{\tau}=0.80 \mathrm{~s}$.

\section{Conclusions}

In this study, we propose a new hydrophone calibration method that does not require a reference hydrophone. It allows correction of the instrument response using a small number of poles and zeros. We applied the method to MERMAID records of the Barcelonnette earthquake $\left(M_{\mathrm{w}} 4.8\right)$, which occurred on 7 April 2014.

The method developed here offers a way to estimate hydrophone responses at low frequencies. It is important to respect the experimental protocol described above to ensure reproducibility of signals. The pressure increase is transmitted to the calibration chamber in a short $(<1 \mathrm{~s})$ but finite time $t_{\tau}$. This results in an input signal somewhat different from a pure step function. This delay time was measured by a second pressure sensor and was estimated to be about $0.73 \mathrm{~s}$. The reasons for this particular value are not clear for the moment because neither the response time of the solenoid valve $(35 \mathrm{~ms})$ nor inertial effects would seem to be able to cause such a relatively large delay. However, it is important to take the delay into account because otherwise the response is clearly underestimated at high frequencies.

The program polezero can be freely downloaded (see Data and Resources). The download includes a user guide, explaining step by step the program functioning for the two considered cases: for underdamped and critical or overdamped signals (two input signals are provided as well).

\section{Data and Resources}

The poles and zeros software (polezero) is available at https://www.geoazur.fr/GLOBALSEIS/Soft.html (last accessed March 2015). Mobile Earthquake Recorder in Marine Areas by Independent Diver (MERMAID) data are collected by Géoazur and available at https://www.geoazur.fr/GLOBALSEIS/Data. html (last accessed March 2015). 


\section{Acknowledgments}

We thank the reviewers and Associate Editor Eric Chael for their fruitful comments that helped improve this article. This work is supported by Osean and the Association Nationale de Recherche et Technique (ANRT). G.N. and A.S. were supported by the European Research Council (ERC, Advanced Grant 226837). A.S. also received financial support from a Centre National de la Recherche Scientifique (CNRS) Chair of Excellence award.

\section{References}

Bobber, R. J. (1988). Underwater Electroacoustic Measurements, Peninsula Press, Los Altos, California.

Giangreco, C. (1997). Mesures acoustiques appliquées aux antennes sonar, Ed., Tech. \& Doc./Lavoisier (in French).

Golenkov, A. N., and L. E. Pavlov (1972). Method of calibrating infrasonic hydrophones, Patent: AD 770520.

Hello, Y., A. Ogé, A. Sukhovich, and G. Nolet (2011). Modern mermaids: New floats image the deep Earth, Eos Trans. AGU 92, 337-338.

Koch, C., and V. Wilkens (2004). Phase calibration of hydrophones: Heterodyne time-delay spectrometry and broadband pulse technique an optical reference hydrophone, J. Phys. Conf. 1, 14-19.

Levin, P. A. (1973). Calibration of Hydrophones, Bruël and Kjaer.

McEachern, J. F. (1984). Very low frequency hydrophone calibration, Patent: 4441173.

Press, W. H., F. B. P. Teukolsky, and W. T. Vettering (1992). Numercial Recipes, Second Ed., Cambridge University Press, Cambridge, United Kingdom.

Radulescu, G., E. A. Lewin, P. J. Wójcik, and A. Nowicki (2003). Calibration of ultrasonic hydrophone probes up to $100 \mathrm{MHz}$ using time gating frequency analysis and finite amplitude waves, Ultrasonics 41, 247-254.

Ringler, A. T., C. R. Hutt, R. Aster, H. Bolton, L. S. D. Gee, and T. Stom (2012). Estimating pole-zero errors in GSN-IRIS/USGS network calibration metadata, Bull. Seismol. Soc. Am. 102, no. 2, 836-841.

Simons, F. J., G. Nolet, J. M. Babcock, R. E. Davis, and J. A. Orcutt (2006). A future for drifting seismic networks, Eos Trans. AGU 87, 31-87.

Simons, F. J., G. Nolet, P. Georgief, J. M. Babcock, L. A. Regier, and R. E. Davis (2009). On the potential of recording earthquakes for global seismic tomography by low-cost autonomous instruments in the oceans, J. Geophys. Res. 114, B05307.
Smith, R. A., and D. R. Bacon (1990). A multiple-frequency hydrophone calibration technique, J. Acoust. Soc. Am. 87, no. 5, 2231-2243.

Veledar, O. (2009). Hydrophone-based calibrator for seawater detection of UHE neutrinos, Nucl. Instrum. Meth. Phys. Res. A 607.

Wessel, P., and W. H. F. Smith (1991). Free software helps map and display data, Eos Trans. AGU 72, 441-446.

Wielandt, E. (2012). Seismometer calibration with program CALEX, in P. Bormann (Editor), New Manual of Seismological Observatory Practice 2 (NMSOP-2), Deutsches GeoForschungsZentrum (GFZ), Postdam, Germany, 1-3.

Zalesak, J. F. (1999). Transfer coupler reciprocity: A new low-frequency coupler-reciprocity technique for the absolute calibration of field hydrophones under full environmental conditions, J. Acoust. Soc. Am. 4, no. 105, 2342-2349.

Osean, ZAE La Bayette

83220 Le Pradet

France

(C.J., J.-F.A.)

Université Nice Sophia Antipolis

CNRS, IRD, Observatoire de la Côte d'Azur

Géoazur UMR 7329

250 rue Albert Einstein

Sophia Antipolis 06560 Valbonne, France

(G.N., A.O., Y.H.)

UMR 6538 Domaines Océaniques

Université Européenne de Bretagne

Université de Bretagne Occidentale

CNRS, IUEM

29280 Plouzané, France

(A.S.)

Manuscript received 28 August 2014;

Published Online 12 May 2015 